Prevention of severe learning disability reviewed by O'Brien $\mathrm{G}$ (Dev Med Child Neurol Aug 2003;45(suppl 95):35-37) emphasizes the detection and early treatment of comorbid autism and ADHD.

\title{
AUTISTIC SPECTRUM DISORDER
}

\section{DIAGNOSIS OF AUTISM}

The identification and assessment process for children with autism and autistic spectrum disorder is reviewed by a developmental pediatrician, speech and language therapist, and consultant in pediatric disability at Guy's and St Thomas' Hospitals, and Great Ormond Street Children's Hospital, London, UK. Autism is a behaviorally defined, neurobiologic disorder and the endpoint of several organic etiologies that include: prenatal insults, metabolic and toxic disorders, tuberous sclerosis, and postnatal encephalitis. A specific medical cause is found in only $6-10 \%$ of cases, most often in those with learning problems. Epilepsy is a common complication. Genetic factors play a key role, twin studies showing a $60 \%$ concordance in monozygotic twins, but no specific candidate genes have been isolated. Most parents identify problems at about 18 months of age, and diagnosis may be made between 2 and 3 years of age. Screening tests can be unreliable. Children with Asperger's syndrome, or 'high functioning' autism, may not be recognized until they are exposed to greater social demands in a school environment. Prevalence of autism is approximately 5 to 6 per 1000 in younger children. The observed increase in the number diagnosed with autism is explained by the recognition of an autistic spectrum rather than a core condition, changes in diagnostic methods, and the inclusion of disorders complicated by autistic symptoms, eg ADHD, Tourette syndrome, and tuberous sclerosis.

In $25-30 \%$ of autistic children, regression of development begins between 15 and 21 months of age. Loss of word use, social withdrawal, loss of eye contact and play interests, unusual behaviors such as flapping are signs of regression. The differential diagnosis includes mental retardation and learning disability, language disorder (aphasias, Landau-Kleffner syndrome), incoordination (developmental coordination disorder), reactive attachment disorder (as in foreign adoptees), epilepsy, Rett syndrome, and neurodegenerative disorders. Prognosis depends on success of interventional therapy, such as cognitive behavior therapy, and social skilis training. IQ, particularly verbal IQ , is a good predictor of future ability to succeed in later life. (Baird G, Cass H, Slonims V. Diagnosis of autism. BMJ 30 August 2003;327:488-493). (Respond: G Baird, Newcomen Centre, Guy's and St Thomas' NHS Trust, London SE1 9RT, UK).

COMMENT. "Autism is melting the edges of the pediatric interface between neurology and psychiatry as they become blended back into one medical discipline" neuropsychiatry, according to Coleman M (Autism: known and unknown. Dev Med Child Neurol Aug 2003;45 (suppl 95):31-34). Autism is not one disease or spectrum but a syndrome and many different diseases. We need to know how and when the pathology is triggered in each subgroup leading to subsequent neurodevelopmental regression. Why is autism predominantly found in males (4:1 ratio)? How do we diagnose autism in the neonate, thus leading to prevention or early therapy? 
Thimerosol and autism. No correlation between thimerosol-containing vaccines and the incidence of autism was found in a Danish population-based study (Madsen KM et al. Pediatrics September 2003;112:604-606). The discontinuation of thimerosol-containing vaccines in Denmark in 1992 was followed by an increase in incidence of autism. A total of 956 children (male-to-female ratio of 3.5:1) were diagnosed with autism during the period from 1971-2000. No trend toward an increase in the incidence of autism occurred during the period when thimerosol was used through 1990. From 1991 to 2000 the incidence of autism increased and continued to rise after the removal of thimerosol, including increases in children born after thimerosol was discontinued.

\section{ENCEPHALOPATHIES}

\section{ENCEPHALOPATHY WITH HASHIMOTO'S THYROIDITIS}

A 14-year-old girl with a 5-year history of hallucinations and depression and a diuagnosis of Hashimoto's thyroiditis is reported from the Mayo Clinic, Rochester, MN. Thyroid-stimulating hormone (TSH) and anti-thyroid antibody titers were significantly elevated. MRI of the brain showed small T2 weighted white matter changes in the frontal lobe, and SPECT scans showed cerebral hypoperfusion deficits. Corticosteroid therapy resulted in significant clinical improvement and resolution of the MRI and temporal lobe SPECT changes. A review of the literature found a total of 16 cases aged 9 to 17 years, all but 2 being female. Thirteen presented with seizures. Only 2 had primarily behavioral presentations, including the Mayo Clinic case. One child, aged 10 years, had longstanding attentional problems, which improved with steroid treatment. Unlike adult patients and the present case, MRI in pediatric cases is usually reported normal. Relapses and residual cognitive deficits may occur. (Mahmud FH, Lteif AN, Renaud DI, Reed AM, Brands CK. Steroid-responsive encephalopathy associated with Hashimoto's thyroiditis in an adolescent with chronic hallucinations and depression: case report and review. Pediatrics September 2003;112:686-690). (Respond: Farid H Mahmud MD, Mayo Clinic, 200 First St SW, Rochester, MN 55905).

COMMENT. Steroid-responsive encephalopathy with Hashimoto's thyroiditis may present with seizures, myoclonus, focal neurologic deficits, and delusions or hailucinations. TSH and thyroid antibody titers are elevated. Patients are usually female and are euthyroid or hypothyroid. Two subtypes are described: 1) vasculitis type with acute stroke-like episodes and focal neurologic deficits and seizures; and 2) diffuse progressive type, with impairment of mental status, confusion, somnolence, and psychosis. Symptoms and MRI changes respond to steroid therapy. The condition was previously named "Hashimoto's encephalopathy."

\section{BICKERSTAFF BRAINSTEM ENCEPHALITIS}

Clinical features of brainstem encephalitis (BBE) in 62 cases and a subgroup with Guillain-Barre syndrome (GBS) are reported from centers in Japan. (Odaka M, Yuki N, Yamada M et al. Brain October 2003;126:2279-2290). BBE and GBS are closely related. 